\title{
Vecchi modi di viaggiare, nuovi modi di parlare? Su camperizzare e camperizzazione
}

\author{
Luisa di Valvasone
}

PUBBLICATO: 31 MARZO 2020

S e ormai da lungo tempo il viaggio in camper accomuna migliaia di appassionati, negli ultimi decenni è diventata una pratica sempre più diffusa - forse anche in contrapposizione alla moda dei camper come "status symbol" - quella di attrezzare e trasformare, secondo il proprio gusto e le proprie possibilità, mezzi di trasporto, come grosse automobili e furgoni, in veri e propri camper; grazie alle loro caratteristiche questi veicoli trasformati permettono di viaggiare in libertà e al tempo stesso di portare con sé lo stretto indispensabile per fermarsi durante il percorso, dormire e magari cucinare qualcosa. Si tratta dunque di una trasformazione che può implicare anche modifiche consistenti, molte aggiunte e ampliamenti della struttura (tetto apribile, cucinotto, bagno). La pratica è già da tempo molto diffusa soprattutto nel nord Europa, ma è in forte aumento anche in Italia, sebbene qui, diversamente da altre nazioni europee, non sia ancora legalmente consentito omologare un cosiddetto furgone camperizzato, chiamato talvolta dagli appassionati del settore anche camper puro (o, più raramente, furgonato). La denominazione di camper puro è usata per distinguere questa variegata tipologia di veicoli dai camper "tradizionali"; una possibile spiegazione - ma in rete si trovano varie discussioni sull'argomento - ci è offerta da un commento pubblicato su un forum dedicato agli appassionati:

[si riporta il testo senza alcuna modifica o correzione] Si usa Chiamarli Camper Puri non perchè gli altri siano impuri (che c'azzecca direbbe Antonino) ma perchè la parola Camper che inizialmante indicava soltanto questa tipologia è stata usurpata anche dalle altre tipologie (mansardati, semintegrali, motorhome) ed è divenuta nel linguaggio comune sinonimo generale di veicolo ricreazionale, per questo si è aggiunto nel tempo il suffisso Puro per distinguerli. (commento di un utente sul forum del sito www.camperonline.it, 24/I2/2007)

La tendenza crescente in fatto di viaggi e turismo all'insegna dell'indipendenza e dello spirito di adattamento sembra aver dato origine anche a nuove formazioni lessicali.

Per le molte segnalazioni del verbo camperizzare giunte alla nostra redazione, non si trovano riscontri in nessun dizionario contemporaneo. Troviamo invece l'aggettivo camperizzato, preceduto dalle voci camper e camperista, nel Supplemento del 2004 al GDLI e nell'edizione del 2007 del GRADIT che lo marca come termine di ambito tecnico-scientifico; entrambi datano la parola al 1993. Di seguito riportiamo la definizione del GDLI seguita dall'attestazione del I993:

Camperizzato, agg. Modificato e adottato [sic, si tratta di un refuso per "adattato"] per venire usato come camper (un furgone).

Business [27-IX-1993], 50: Transit camperizzato vetrato nell'8I vendo a L 2.500.00o non trattabili.

$=$ Deriv. da camper coi suff. dei verbi frequent. e dei part. pass.

Come si legge sia nel GDLI sia nel GRADIT, camperizzato deriva dal sostantivo invariabile camper 'furgone internamente attrezzato per l'abitazione in modo analogo a una roulotte, usato per turismo' (GDLI), a sua volta giunto dall'inglese camper, propriamente 'campeggiatore', derivato dal verbo to camp 'accamparsi, campeggiare', e presente nella nostra lingua a partire, secondo il GRADIT e il 
GDLI, dal 1976. Sebbene i dizionari segnalino come data di prima attestazione il 1993, la ricerca negli archivi online dei quotidiani consente di rintracciare attestazioni anteriori dell'aggettivo, e dunque di retrodatarlo; nell'archivio del "Corriere della Sera" infatti l'aggettivo (declinato in tutte le sue forme) compare in 7 articoli, il primo dei quali risale al ı88:

È richiesto un gruppo di almeno dieci persone. Si viaggia con una Land Rover Iog e con un camion Mercedes Unimog camperizzato. (Franco Damerini, Sahara: in fuoristrada e cammello alla grande festa dei Tuareg, "Corriere della Sera", I6/5/198I)

Se camper, camperista ('turista che viaggia in camper', Vocabolario Treccani online) e camperizzato sono stati accolti dagli strumenti lessicografici - sebbene camperizzato non compaia in nessun altro dizionario oltre quelli già citati -, il verbo camperizzare non è invece presente in nessun vocabolario contemporaneo.

La prima attestazione di camperizzare che siamo riusciti a rintracciare in rete, nello specifico in un blog di viaggi, è datata 2003, sebbene si riferisca a un'esperienza personale dell'autore risalente agli anni Ottanta:

Nel 1980 riuscii ad acquistare e camperizzare un furgone $\mathrm{UAZ}_{4 \times 4}$, realizzando un altro sogno: tornare nella "mia Africa" con la "mia casa". (Mal d'Africa/2 - Algeria, sul sito pleinair.it, zo dicembre 2003)

Questo dato porta a supporre che il verbo possa essere stato ricostruito sull'aggettivo camperizzato interpretato come un participio passato; quest'ultimo infatti, oltre a essere anteriore di quasi un ventennio, risulta ben attestato nei quotidiani e in rete (tra le pagine in italiano di Google, il $24 / 3 / 2020$, emergono in totale 73.040 ) e si ritrova frequentemente nel sintagma furgone camperizzato, anche se si parla, in minor misura, anche di camion camperizzato, auto/macchina/roulotte camperizzata, van o minivan camperizzati, oltre naturalmente al frequente uso dei nomi propri delle auto e dei furgoni (Opel Zafira camperizzata, Fiat Ducato camperizzato). Sulla base di camperizzato, da camper + -izzato, si sarebbe formato il verbo camperizzare, e non viceversa; il verbo sembra dunque posteriore all'aggettivo e, come vedremo in seguito, anche al sostantivo camperizzazione. A proposito delle retroformazioni nell'italiano, nell'Enciclopedia dellitaliano (20II) presente sul sito Treccani.it, Claudio Iacobini segnala che l'attestazione di "verbi regolari in -izzare successiva a quella di derivati nominali in -izzazione (per es., tindalizzare I942; tindalizzazione I9I9) [...] è di solito interpretata come la realizzazione di una fase intermedia (rimasta virtuale per un certo periodo di tempo) di un processo additivo di formazione delle parole secondo il normale iter derivazionale, e non come esempio di retroformazione". Per approfondimenti sulla produttività del suffisso -izzare nella formazione di verbi prevalentemente transitivi rimandiamo alla scheda pubblicata sul nostro sito.

Per quanto riguarda l'attuale diffusione del verbo, si rintraccia un discreto numero di attestazioni in rete. Esistono infatti moltissimi blog che si occupano dell'argomento, forum dedicati agli appassionati e siti, anche di professionisti, che vendono furgoni camperizzati o si offrono di camperizzare il vostro veicolo. Tra le pagine in italiano di Google, il 24/3/2020 emergono 9.370 risultati su Google per la forma all'infinito, mentre per le forme flesse abbiamo, ad esempio, 266 risultati per camperizzo, I3I per camperizziamo, I3I per camperizzano, I96 per ho camperizzato, I69 per abbiamo camperizzato, 86 per hanno camperizzato, 366 per camperizzando. Nettamente inferiori sono le occorrenze su Google Libri: solo 2 risultati effettivi per la forma all'infinito del verbo (a partire dal 2004) e rare le forme flesse.

La ricerca sui quotidiani restituisce invece pochissime attestazioni (mentre se ne hanno diverse per l'aggettivo camperizzato). Segnaliamo un solo articolo della "Stampa" pubblicato nel 2019 dal titolo 
Tutti pazzi per la vacanza in camper. Ecco come si fa la "van conversion" (https://www.lastampa.it/motori/attualita/2019/o7/21/news/tutti-pazzi-per-la-vacanza-in-camperecco-come-si-fa-la-van-conversion-I.37150374); non si trovano altre attestazioni, né della forma all'infinito né delle forme flesse, nell'archivio della "Stampa" (che raccoglie articoli dal i867 al 2006) e in quello del "Corriere" (dal I876 ad oggi). Nell'archivio della "Repubblica" non emergono risultati per la forma camperizzare né per alcune forme flesse ricercate come camperizzo, camperizza, camperizzano, camperizzarono, camperizzando, ma troviamo un'attestazione per hanno camperizzato e una per abbiamo camperizzato, entrambe poste tra virgolette, segno che la parola è vista come propria di un gergo, in parte ironica in parte identitaria, o comunque non ancora percepita come pienamente acclimatata nel nostro lessico:

Hanno fatto una scelta sette anni fa: basta pagare affitti e bollette. Davide ha comprato un camion, insieme a Francesca lo hanno "camperizzato" e ne hanno fatto la loro casa. Mobile. Dal 2009 vivono in viaggio e regalano il cinema agli abitanti dei villaggi più isolati del mondo. (Giulia Echites, "Cinema du Desert", due italiani itineranti: "Con i film portiamo tanta felicità", LaRepubblica.it, 8/3/2016)

"[...] E poi, naturalmente, abbiamo "camperizzato" gli interni perché Piero, questo il nome del nostro Subaru, deve ospitarci per un intero mese". (Ilaria Brugnotti, Mongol Rally, la sfida dei quattro ingegneri della Maserati, LaRepubblica.it, sez. "Motori", го/7/2018)

Accanto a camperizzare si rintracciano rare occorrenze della variante camperare: questo verbo, derivato anch'esso dall'inglese camper, ha tuttavia maggior successo nel gergo dei giochi di ruolo online dove viene largamente impiegato con valore intransitivo e con altro significato, e cioè per indicare l'azione che compie un personaggio che staziona in un luogo con pazienza aspettando i nemici da uccidere'.

Come già accennato, oltre al verbo camperizzare si registra l'uso del sostantivo camperizzazione, per indicare la pratica di camperizzare un veicolo, a fianco dell'inglese van conversion, talvolta tradotto in conversione van. Il $24 / 3 / 2020$ tra le pagine in italiano di Google troviamo II.70o risultati per camperizzazione, ai quali possiamo aggiungerne $35^{2}$ per il plurale camperizzazioni. Il sostantivo si mostra comunque meno diffuso del concorrente van conversion, forestierismo non adattato per cui si contano 27.300 risultati. Su Google Libri troviamo invece 2 occorrenze di camperizzazione (e 2 per il plurale) di cui la prima datata 1990 in un articolo sull'"Espresso" a proposito dei termini camper e roulotte, scritto da Tullio De Mauro (non è stato possibile recuperare l'esatta edizione e dunque il mese):

Il vocabolo d'origine francese [roulotte] è stato finora assai meglio insediato nel nostro lessico, come provano i numerosi derivati (roulottista, roulottopoli, roulottizzare). Al confronto, il vocabolo d’origine inglese [camper], d'acquisto più recente, resta ancora più marginale: se si può sospettare che qua e là camperista sia già in uso, di camperizzazione e camperopoli non si è ancora parlato. Almeno, mentre scrivo. (Tullio de Mauro, La parola della settimana, "L'Espresso", edizioni I4-20, I990, p.23)

Nel passo appena riportato camperizzazione e camperopoli sono neoformazioni solo ipotizzate da Tullio De Mauro, mentre la parola di origine francese roulotte, stando alla citazione, aveva prodotto derivati già dagli anni Novanta; dal sostantivo roulotte si è formato il verbo roulottizzare, il quale tuttavia non è presente nei dizionari contemporanei. Troviamo invece sia roulottista ('chi viaggia, campeggia o vive in roulotte', ZINGARELLI 2020), che il GRADIT data al I958, sia roulottopoli ('agglomerato di roulotte installate su un terreno in prossimità di un centro abitato colpito da una calamità naturale, per accogliere gli abitanti che abbiano dovuto abbandonare le loro case o per ospitare profughi, senzatetto e sim.', ZINGARELLI 2020), attestato secondo il GRADIT a partire dal 1983.

Tornando alla nostra analisi, De Mauro ipotizzava camperizzazione come una forma possibile ma non 
ancora in uso nel I990; sono tuttavia da segnalare alcune precedenti, sporadiche attestazioni nell'archivio della "Stampa" in cui troviamo 2 risultati per il singolare e 2 per il plurale tutti precedenti agli anni Novanta, il primo dei quali risale al 1979 in un annuncio di vendita:

VENDESI autocarri Mercedes 4X4 [non leggibile] 404 con progetto per camperizzazione Telefonare XXX. (annuncio di vendita privati, "La Stampa", 22/7/1979)

Le altre occorrenze riguardano due identiche inserzioni pubblicitarie di un'officina ("camperizzazioni totali o parziali, fisse o mobili, su qualsiasi furgone nuovo o usato nostro o vostro"), pubblicate nel I982 e nel I983, e un articolo di cronaca nera del I983 nel quale si riporta la notizia dell'omicidio del titolare di unofficina ("Titolare della piccola industria di camperizzazione e costruzione rimorchi da campeggio"): si tratta dunque di professionisti che evidentemente si occupavano già in quegli anni di camperizzazioni. Tuttavia, le occorrenze emerse dall'archivio della "Stampa", sebbene forniscano la prima attestazione del sostantivo, rappresentano un caso isolato. Nell'archivio del "Corriere" troviamo ununica occorrenza, datata I982, la quale tuttavia, come si evince dal contesto, ha una diversa accezione rispetto a quella che a noi interessa, riferita alla trasformazione di aree pubbliche o private in campeggi e aree di sosta per camper e roulotte:

Una battuta d'arresto sembra registrarsi nella corsa verso la «camperizzazione»: crescente in questi anni anche per la massiccia pubblicità, l'acquisto di camper, roulotte e tende comincia a trovare ostacoli nelle difficoltà di reperire campeggi e piazzole. (Victor Ciuffa, Su molte spiagge l'afflusso dei turisti è già quello del pienone di Ferragosto, "Corriere della Sera", 25/7/1982)

Tale accezione sembra però non aver avuto successo nella nostra lingua: sebbene si ritrovi in un articolo della "Repubblica" pubblicato nel 2007 (unica attestazione del sostantivo presente nell'archivio), i risultati che emergono dalla ricerca in rete riguardano esclusivamente, almeno per quanto siamo riusciti a verificare, la trasformazione e l'allestimento di furgoni e auto. In ogni caso, oltre all'attestazione di De Mauro e a quelle anteriori trovate nell'archivio della "Stampa", le occorrenze rintracciabili in rete di camperizzazione, nell'accezione che a noi interessa, si hanno a partire dal Duemila; la prima risale al 200I, in un commento di un utente in un forum dedicato al "turismo itinerante":

Salve, sono il fortunato possessore di un IVECO Daily td 4x4, con trazione integrale, ridotte e blocco differenziale posteriore, camperizzato in alluminio dalla ditta Kelber (che ora non esiste più). Telaio passo 3.200, pneumatici 7.50-16 stradali oppure 9.00-16 da fuoristrada, ricavato da un mezzo allestito cassone. La camperizzazione è stata fatta in alluminio. Ha già fatto 3 volte la libia, l'Iran, l'Islanda e capo Nord cavandosela egregiamente. (dal sito turismoitinerante.com, I2/7/200I)

Al di là delle attestazioni, quel che è certo è che la pratica della camperizzazione iniziò a diffondersi in Italia già dalla fine degli anni Settanta. È dunque plausibile che, come camperizzato e camperizzazione, anche camperizzare circolasse limitatamente nel gergo del gruppo ristretto di appassionati di questo tipo di turismo. Negli anni successivi la camperizzazione è diventata in Italia una pratica sempre più diffusa e specializzata, e gli interventi di trasformazione di un furgone, o simili, in un camper puro possono oggi essere molto ingenti, oltre che dispendiosi; con l'aumentare degli appassionati e la nascita di professionisti del settore, è aumentato anche l'uso dei termini collegati alla pratica stessa. Il fatto che in Italia non sia ancora legalmente consentita l'omologazione di furgoni camperizzati potrebbe essere una delle ragioni per cui le parole collegate a questa pratica circolano soprattutto in contesti informali, molto meno in quelli di media formalità come i quotidiani, e niente affatto in testi ufficiali come leggi e regolamenti. Sarà il tempo a dirci quali "viaggi" percorreranno queste parole. 


\section{Cita come:}

Luisa di Valvasone, Vecchi modi di viaggiare, nuovi modi di parlare? Su camperizzare $e$ camperizzazione , "Italiano digitale", 2020, XII, 2020/1 (gennaio-marzo)

DOI: $10.35948 / 2532-9006 / 2020.3313$

Copyright 2020 Accademia della Crusca

Pubblicato con licenza creative commons CC BY-NC-ND 